OPEN ACCESS

Edited by:

Yan Zou,

Henan University, China

Reviewed by:

Axel Montagne,

University of Edinburgh,

United Kingdom

Virginia Pensabene,

University of Leeds, United Kingdom

*Correspondence:

Juntao Wang

juntao-wang@hotmail.com

Xiaoming Zhu

495072782@qq.com

Jia Li

jia.li1@mq.edu.au

Specialty section:

This article was submitted to

Nanobiotechnology,

a section of the journal

Frontiers in Bioengineering and

Biotechnology

Received: 27 February 2021

Accepted: 26 April 2021

Published: 11 June 2021

Citation:

Wang J, Xu F, Zhu X, Li X, Li Y and Li J (2021) Targeting microRNAs

to Regulate the Integrity of the

Blood-Brain Barrier.

Front. Bioeng. Biotechnol. 9:673415.

doi: 10.3389/fbioe.2021.673415

\section{Targeting microRNAs to Regulate the Integrity of the Blood-Brain Barrier}

\author{
Juntao Wang ${ }^{1,2 *}$, Fang $X u^{1,2}$, Xiaoming Zhu ${ }^{1,2 *}$, Xianghua $L i^{3}$, Yankun $L i^{3,4}$ and Jia $L i^{5 *}$ \\ 'School of Nuclear Technology and Chemistry and Biology, Hubei University of Science and Technology, Xianning, China, \\ ${ }^{2}$ Hubei Key Laboratory of Radiation Chemistry and Functional Materials, Hubei University of Science and Technology, \\ Xianning, China, ${ }^{3}$ School of Pharmacy, Hubei University of Science and Technology, Xianning, China, ${ }^{4}$ Hubei Key Laboratory \\ of Cardiovascular, Cerebrovascular, and Metabolic Disorders, Hubei University of Science and Technology, Xianning, China, \\ ${ }^{5}$ Centre for Motor Neuron Disease, Department of Biomedical Sciences, Faculty of Medicine and Health Sciences, \\ Macquarie University, Sydney, NSW, Australia
}

The blood-brain barrier (BBB) is a highly specialized neurovascular unit that protects the brain from potentially harmful substances. In addition, the BBB also engages in the exchange of essential nutrients between the vasculature and brain parenchyma, which is critical for brain homeostasis. Brain diseases, including neurological disorders and cerebrovascular diseases, are often associated with disrupted BBB integrity, evidenced by increased permeability. Therefore, defining the mechanisms underlying the regulation of BBB integrity is crucial for the development of novel therapeutics targeting brain diseases. MicroRNAs (miRNA), a type of small non-coding RNAs, are emerging as an important regulator of BBB integrity. Here we review recent developments related to the role of miRNAs in regulating BBB integrity.

Keywords: blood-brain barrier, regulate, targeting, microRNA, nanobiotechnology

\section{INTRODUCTION}

The blood-brain barrier (BBB) is a multicellular neurovascular complex, mainly consisting of brain endothelial cells and supporting cells, such as pericytes and astrocytes (Profaci et al., 2020). In addition to its key role in preventing neurotoxic agents from entering the brain, the BBB also regulates the exchange of essential nutrition between the brain and the blood (Banks, 2016). The function of the BBB depends on its intact structure, or BBB integrity (Banks, 2016). Disrupted $\mathrm{BBB}$ integrity has been shown to contribute to the onset and progression of diseases in the brain, including neurodegenerative diseases and cerebrovascular diseases (Sweeney et al., 2018).

A large body of studies has focused on understanding how BBB integrity is regulated (Sweeney et al., 2016; Nation et al., 2019; Li et al., 2020). These efforts have led to the finding of several key molecules and singling pathways, in both brain endothelial cells and supporting cells, which are critical for the maintenance of BBB integrity, including microRNAs (miRNAs), endothelial junction molecules (e.g., VE-cadherin, claudin-5), fatty acid transporter (e.g., mfsd2a), platelet-derived growth factor (PDGF) signaling, and Wnt/ $\beta$-catenin signaling (Ben-Zvi et al., 2014; Chakraborty et al., 2020; Li et al., 2020).

MicroRNAs are a class of endogenous small non-coding RNAs (20-25 nucleotides) that regulate genes at the post-transcriptional stage through either cleavage of mRNA or inhibition of translation (Chakraborty et al., 2020). In humans, approximately 2,500 mature miRNAs have been identified to regulate more than $30 \%$ of all proteins expressed in humans, suggesting the profound role of miRNAs in human physiology and pathology (Kozomara et al., 2019). Recently, the role of miRNAs 
in the modulation of $\mathrm{BBB}$ integrity has drawn great attention (Toyama et al., 2018; Chakraborty et al., 2020). In this review, we attempt to outline the involvement of miRNAs in the regulation of BBB integrity. We first briefly summarize the biogenesis and function of miRNAs, followed by a discussion of how miRNAs regulate $\mathrm{BBB}$ integrity by targeting different units in the $\mathrm{BBB}$ complex with an emphasis on the brain endothelial cells. We end by highlighting the challenges of developing efficient miRNAbased therapeutics targeting the disrupted BBB.

\section{BIOGENESIS AND FUNCTION OF miRNAs}

MicroRNA genes, which can be either intergenic or intronic, are first transcribed by RNA polymerase II to pri-miRNAs, followed by being processed into pre-miRNAs in the nucleus (Treiber et al., 2019). Pre-miRNAs are then exported to the cytoplasm to be further processed into imperfect double-stranded RNA duplex including guide strand (miRNA) and passenger strand (miRNA*). After being loaded to the RNA-induced silencing complex (RISC), the passenger strand is quickly excluded from RISC and degraded rapidly, leading to a strong preference toward the guide stand, the form of a mature miRNA (Gebert and MacRae, 2019).

MicroRNA is a posttranscriptional regulator of gene expression that contributes to diverse cellular processes, such as development, proliferation, differentiation, and apoptosis. MiRNAs recognize and bind to their target mRNAs via the seed region, a sequence of six contiguous nucleotides found from position 2-7 at the $5^{\prime}$-end of the molecule through direct Watson-Crick base-pairing (Gebert and MacRae, 2019). Once loaded into the RISC, the mature miRNA acts to guide the RISC to bind to partially complementary sequences within the $3^{\prime}$ untranslated region (UTR) of target mRNAs, resulting in the destabilization of mRNA or/and inhibition of translation (Treiber et al., 2019). For example, the first miRNA, lin-4, negatively regulates its target, lin-14, by repressing translation. In comparison, miR-27a regulates its target VE-cadherin at both mRNA and protein level (Young et al., 2013). It has been proposed that ancillary nucleotides at the $3^{\prime}$-end of the miRNA also play an important role in target recognition (Li et al., 2016).

\section{MiRNAs IN REGULATING BBB INTEGRITY}

The integrity of the $\mathrm{BBB}$ is mainly determined by brain endothelial cells, which are the fundamental unit of the BBB (Greene et al., 2020). Changes in tight junctions between brain endothelial cells and transcytosis in these cells have a significant effect on BBB integrity (Ayloo and Gu, 2019). In addition, crosstalk between endothelial cells and supporting cells forming the neurovascular unit (NVU), such as pericytes, astrocytes, immune cells, and other cells in the brain also contributes to the maintenance of BBB integrity (Profaci et al., 2020). Despite the fact that miRNAs are involved in brain diseases through various pathways (Juzwik et al., 2019; Ludwig et al., 2019; Sonoda et al., 2019; Starhof et al., 2019; Qian et al., 2020), the majority of miRNAs found to regulate $\mathrm{BBB}$ integrity exert their impact by targeting brain endothelial cells (Chakraborty et al., 2020). MiRNAs can either directly target endothelial junction molecules or modulate inflammation, endothelial cell survival, apoptosis, actin cytoskeleton, and other pathways to indirectly influence tight junctions in the BBB, leading to change in BBB integrity. Additionally, miRNAs may also have an impact on the crosstalk between brain endothelial cells and supporting cells, which is critical for the maintenance of BBB integrity (Figure 1).

\section{MiRNAs and Tight Junctions}

One of the key features of the BBB is the existence of extremely tight junctions between brain endothelial cells (Vanlandewijck et al., 2018), which are controlled by a range of junction molecules, including tight junction molecules [e.g., zona occludens (ZO), occludin, and claudin-5] and adherens junction molecules (e.g., VE-cadherin) (Cristante et al., 2013). MiRNAs have been shown to directly target these junction molecules, leading to a change in BBB integrity (Ma et al., 2017, 2020; Zuo et al., 2019; Table 1).

During ischemic stroke, BBB integrity is compromised as shown by increased immune cell infiltration and solute leak, eventually leading to neuronal loss. In ischemic stroke, miR$15 \mathrm{a} / 16$-1 cluster was significantly upregulated to mediate BBB breakdown by direct downregulation of claudin-5. The specific depletion of the miR-15a/16-1 cluster in endothelial cells enhanced brain claudin- 5 expression after transient ischemia in mice, resulting in the restoration of $\mathrm{BBB}$ integrity with smaller brain infarcts and decreased neuroinflammation (Ma et al., 2020). ZO-1 is another highly expressed tight junction molecule in the BBB Toyama et al. (2018). Identified miR-501-3p mediated inflammation-induced $\mathrm{BBB}$ breakdown via directly targeting $\mathrm{ZO}$ 1. Using a mouse model of vascular cognitive impairment with increased inflammation, they further showed that the expression of miR-501-3p and its target ZO1 were inversely correlated. Inhibition of miR-501-3p with a specific inhibitor rescued ZO1 gene expression, leading to restoration of BBB integrity within the white matter and amelioration of working memory deficits.

The regulation of tight junctions by miRNA can also be indirect. For example, miRNAs can control the transcription factor of tight junction molecules to regulate their expression. One of the transcription factors of claudin-5 is forkhead box protein $\mathrm{O} 1$ (FoxO1), which positively regulates claudin-5 expression (Taddei et al., 2008). By directly targeting FoxO1, miR182 negatively regulates claudin-5 expression and tight junctions of brain endothelial cells, while inhibition of miR-182 protects BBB integrity (Zhang et al., 2020). MiR-107 was identified to directly target endophilin-1 (Liu et al., 2016), which regulates ZO-1 and occludin expression via the epidermal growth factor receptor (EGFR)-extracellular signal-regulated protein kinase (ERK)1/2 pathway (Liu et al., 2014). In addition, miR-143 was shown to contribute to methamphetamine-induced BBB disruption by targeting p53 unregulated modulator of apoptosis (PUMA), which leads to a decrease of tight junction molecules, such as claudin-5, occludin, and ZO-1 (Bai et al., 2016). 


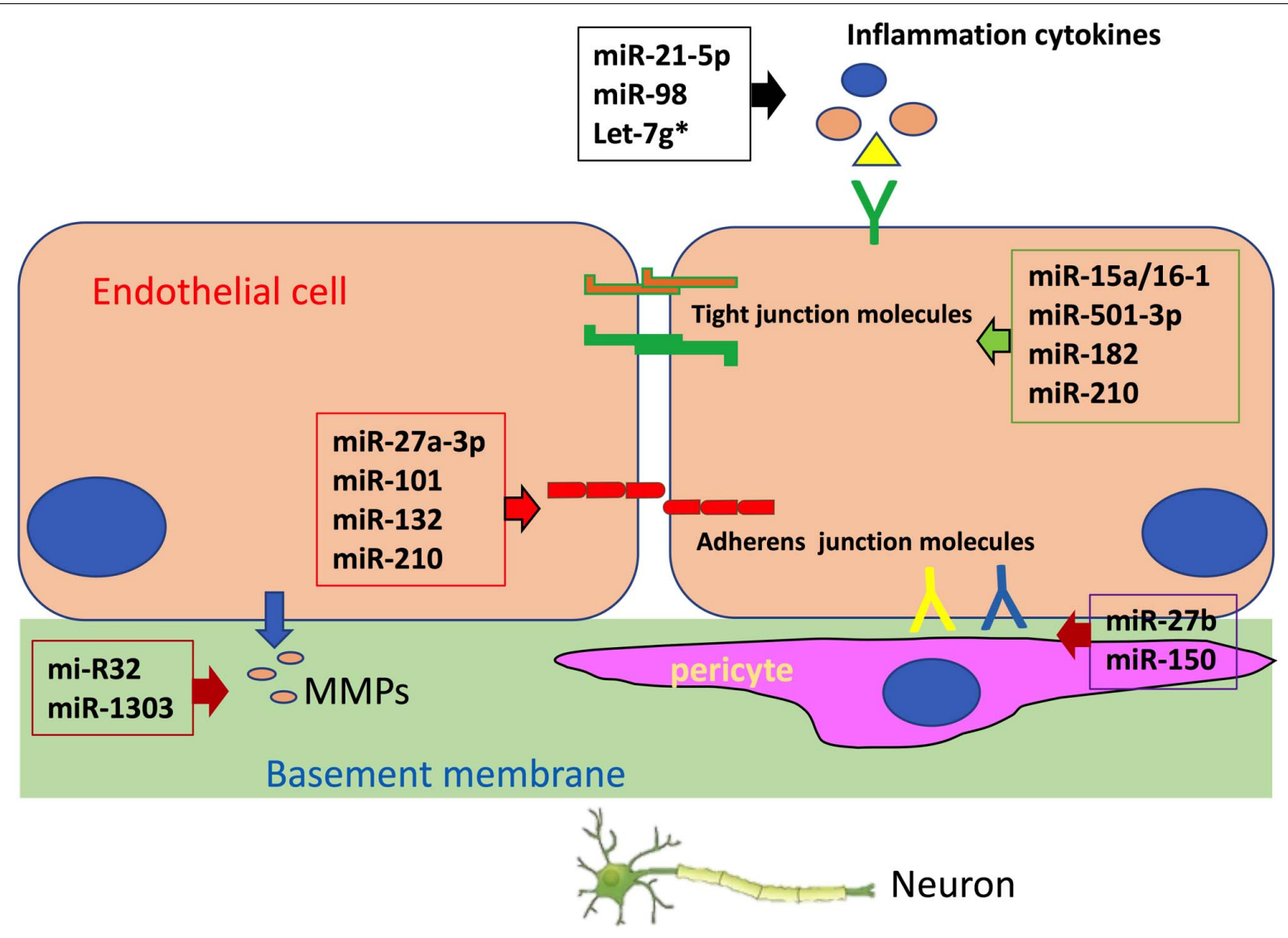

FIGURE 1 | MicroRNAs (miRNAs) regulate blood-brain barrier (BBB) integrity by targeting endothelial tight junction, basement membrane, pericyte coverage, and inflammation.

Adherens junctions form before tight junctions and are also a major regulator in vascular integrity (Taddei et al., 2008; Lampugnani et al., 2018). VE-cadherin, a key adherens junction molecule, was shown to be decreased in cerebral vascular diseases (Li et al., 2020) and neurodegenerative diseases (Li et al., 2018). MiR-27a directly regulates VE-cadherin (Young et al., 2013). In cerebral cavernous malformation, where BBB is disrupted, blockage of the interaction between miR-27a and VE-cadherin by a specific target site blocker CD5-2 restored BBB integrity and reduced severity of diseases (Li et al., 2020). VE-cadherin was also shown to be regulated by miR-101 (Mishra and Singh, 2013). In HIV-1-infected human brain microvascular endothelial cells, miR-101 mediated the disruptive effect of infection on endothelial barrier integrity by downregulating VE-cadherin. MiRNA may also indirectly regulate VE-cadherin. For example, miR-132 directly targets eukaryotic elongation factor 2 kinase (EEF2K), which inhibits VE-cadherin by phosphorylation of eukaryotic elongation factor 2 (eEF2) (Xu et al., 2017). In this interesting study, neurons secreted miR-132-containing exosomes to brain endothelial cells, leading to an increase in functional and mature miR-132 expression in brain endothelial cells. Consequently, VE-cadherin was upregulated, and BBB integrity was enhanced. When miR-132 was antagonized by specific miR-132 morpholino antisense oligonucleotides, severe intracranial hemorrhage and disrupted $\mathrm{BBB}$ integrity was exhibited. Although VE-cadherin is an upstream regulator of claudin-5 and tight junctions (Taddei et al., 2008), in this study, changes in tight junction molecules such as claudin-5, occludin, ZO-1, were not observed, suggesting VE-cadherin can modulate $\mathrm{BBB}$ integrity via modulating adherens junctions without altering tight junctions.

Some miRNAs regulate BBB integrity by targeting both tight and adherens junction molecules. For example, miR-210 was shown to directly regulate tight junction molecule occludin and adherens junction molecule $\beta$-catenin (Ma et al., 2017). During ischemia, miR-210 expression was significantly upregulated in the brain. Inhibition of miR-210 with its complementary locked nucleic acid oligonucleotides (miR-210-LNA) reduced BBB leakiness by increasing expression of both occludin and $\beta$-catenin in the BBB.

\section{MiRNAs and Inflammation}

Inflammation is often associated with disrupted BBB (Haruwaka et al., 2019). On the one hand, miRNAs can mediate inflammation-induced BBB permeability; on the other hand, miRNAs may act as an upstream of inflammation by targeting inflammation molecules and pathways to regulate BBB integrity (Table 1). 
TABLE 1 | The effect of microRNAs (miRNAs) on blood-brain barrier (BBB) integrity.

\begin{tabular}{|c|c|c|c|c|}
\hline Modulation of the BBB & miRNA & Target & Function & References \\
\hline \multirow[t]{9}{*}{ Tight junction } & $\operatorname{miR}-15 a / 16-1$ & Claudin-5 & BBB destructive & Ma et al., 2020 \\
\hline & miR-501-3p & ZO1 & BBB destructive & Toyama et al., 2018 \\
\hline & miR-182 & FoxO1 & BBB destructive & Taddei et al., 2008 \\
\hline & miRNA-107 & Endophilin-1 & BBB destructive & Zhang et al., 2020 \\
\hline & miR-143 & PUMA & BBB destructive & Bai et al., 2016 \\
\hline & miR-27a-3p & VE-cadherin & BBB destructive & Li et al., 2020 \\
\hline & miR-101 & VE-cadherin & BBB destructive & Mishra and Singh, 2013 \\
\hline & miR-132 & EEF2K & BBB protective & Xu et al., 2017 \\
\hline & $\operatorname{miR}-210$ & Occludin $/ \beta$-catenin & BBB destructive & Ma et al., 2017 \\
\hline \multirow[t]{7}{*}{ Inflammation } & $\operatorname{miR}-125 a-5 p$ & N/A & BBB protective & Reijerkerk et al., 2013 \\
\hline & miR-155 & $\mathrm{N} / \mathrm{A}$ & BBB destructive & Lopez-Ramirez et al., 2014 \\
\hline & $\operatorname{miR}-21-5 p$ & TNF- $\alpha / \mathrm{IL}-6$ & BBB protective & Ge et al., 2016 \\
\hline & miR-126-3p & VCAM-1 & BBB protective & Fu et al., 2019 \\
\hline & miR-98 and let- $7 g^{\star}$ & CCL2 and CCL5 & BBB protective & Rom et al., 2015 \\
\hline & $\operatorname{miR}-1303$ & MMP9 & BBB protective & Lampugnani et al., 2018 \\
\hline & miR-132 & MMP9 & BBB protective & Li et al., 2018 \\
\hline \multirow[t]{2}{*}{ Supporting cells coverage } & miR-27b & SEMA6A/D & BBB protective & Demolli et al., 2017 \\
\hline & miR-150 & Tie-2 & BBB destructive & Fang et al., 2016 \\
\hline \multirow[t]{2}{*}{ Apoptosis/cell cycle } & miR-182 & mTOR & BBB protective & Zhang et al., 2020 \\
\hline & miR-285 & Yki/Mask & BBB protective & Li et al., 2017 \\
\hline Actin cytoskeleton & miRNA-181c & PDPK1 & BBB destructive & Tominaga et al., 2015 \\
\hline \multirow[t]{2}{*}{ Channel and receptor } & miR-30a & $\mathrm{ZnT4}$ & BBB destructive & Wang et al., 2021 \\
\hline & miR-27a-3p & AQP11 & BBB protective & Xi et al., 2018 \\
\hline
\end{tabular}

In brain endothelial cells treated with the proinflammatory mediator tumor necrosis factor $\alpha /$ interferon $\gamma$ (TNF $\alpha / \mathrm{IFN} \gamma)$, 107 miRNAs were significantly changed (Reijerkerk et al., 2013), among which miR-125a-5p was downregulated. Consistently, in the inflamed blood vessels of patients with multiple sclerosis (MS), there was also significantly less miR-125a-5p than in its expression in non-inflamed blood vessels (Reijerkerk et al., 2013), suggesting a positive correlation between miR-125a-5p and $\mathrm{BBB}$ integrity during inflammation. In contrast, miR-155 was shown to negatively affect $\mathrm{BBB}$ integrity during inflammation (Lopez-Ramirez et al., 2014). It was upregulated in disrupted BBB of MS human patients and animals-in experimental allergic encephalomyelitis (EAE), a model of MS. When miR155 was knocked out in EAE mice, BBB leakage was reduced by $50 \%$ compared with wild-type mice. Inhibition of miR155 also reduced TNF $\alpha / \mathrm{IFN} \gamma$-induced endothelial permeability in vitro. The putative targets of miR- 155 included focal adhesion molecules and junctional complex, suggesting miR-155 may function by targeting these molecules to modulate BBB integrity.

MicroRNAs can also target inflammatory cytokines or markers to regulate BBB integrity, as exemplified by miR-21$5 \mathrm{p}$, which regulates the BBB by targeting pro-inflammatory cytokines TNF- $\alpha$, interleukin 6 (IL-6), and nuclear factor kappa B (NF-кB) signaling (Ge et al., 2016). Another miRNA, miR126 , was shown to attenuate intracerebral hemorrhage-induced leukocyte adhesion and BBB disruption by targeting vascular cell adhesion molecule-1 (VCAM-1), a classic inflammation marker critical for leukocyte adhesion to blood vessels (Fu et al., 2019). Glycogen synthase kinase $3 \beta$ (GSK3 $\beta$ ) was shown to protect BBB under neuro-inflammation conditions. MiR-98 and let- $7 \mathrm{~g}^{*}$, both of which belong to the highly conserved let-7 family, mediated the BBB-protective effect of GSK3 $\beta$ by targeting inflammatory molecules CCL2 and CCL5 (Rom et al., 2015). Overexpression of let- $7 \mathrm{~g}^{*}$ and miR-98 reduced neuro-inflammation-induced BBB leakiness. Matrix metalloproteinase-9 (MMP9) contributes to inflammation-induced BBB breakdown (Shigemori et al., 2006; Turner and Sharp, 2016). Several miRNAs, including miR-1303 and miR-132, have been shown to target MMP9 and play a protective role in BBB integrity under inflammation conditions (Song et al., 2018; Zuo et al., 2019).

\section{MiRNAs and Crosstalk Between Brain Endothelial Cells and Supporting Cells}

Pericyte coverage on brain endothelial cells is another key indicator of BBB integrity (Ting et al., 2019). When pericyte coverage is reduced, BBB permeability is increased. MiRNAs can modulate the integrity of the BBB by regulating pericyte coverage-associated molecules. One of the examples is miR-27. There are two miR-27s, miR-27a and miR-27b, which differ from each other by one nucleotide outside the seed region (Young et al., 2013). While miR-27a targets VE-cadherin to compromise BBB integrity, miR-27b promotes the interaction of endothelial cells with pericytes by targeting semaphorin 6A/D (SEMA6A/D), leading to enhancement of endothelial barrier function (Demolli et al., 2017; Table 1). This opposite role of miRNAs with identical seed regions is not rare, as evidenced by the difference in 
miR-23a and miR-23b (Li et al., 2016), reflecting the complexity of miRNA regulation.

The recruitment of pericytes to the endothelium can also be mediated by angiopoietins/Tie-2 signaling. MiR-150 was shown to target Tie-2 (Fang et al., 2016), leading to inhibition of claudin-5 expression and endothelial cell survival. It will be interesting to see whether miR-150 also has an effect on pericyte coverage to the $\mathrm{BBB}$. Astrocytes, another type of $\mathrm{BBB}$ supporting cells, can release factors to strengthen $\mathrm{BBB}$ function via regulating miRNAs, one of which is mir-125a-5p, in the brain endothelial cells. How these miRNAs mediate crosstalk between astrocytes and brain endothelial cells remains to be defined (Reijerkerk et al., 2013).

\section{MiRNA and Other BBB-Relevant Pathways}

In addition to the aforementioned endothelial tight junctions, inflammation and supporting cell coverage, other pathways regulating endothelial function, such as cell survival, cytoskeleton, and ion channels also contribute to the regulation of BBB integrity (Table 1).

Endothelial cell survival is critical for its function. MiR-182 was shown to mediate $\mathrm{BBB}$ breakdown in cerebral ischemia, a disease associated with massive $\mathrm{BBB}$ damage. Further studies suggested that miR-182 directly targeted mTOR, which is antiapoptotic, suggesting miR-182 may regulate $\mathrm{BBB}$ integrity by regulating apoptosis of brain endothelial cells after ischemia. Mir285 is another mRNA shown to affect BBB integrity through regulating apoptosis (Li et al., 2017). By targeting Yorkie (Yki in Drosophila, or YAP and TAZ in mammals), miR-285 inhibits cell proliferation and induces apoptosis, leading to regulation of BBB integrity.

The actin cytoskeleton is associated with endothelial barrier functions (Nag, 1995), through intercellular connections with tight and adherens junctions between endothelial cells. MiR181C was shown to target 3-phosphoinositide-dependent protein kinase-1 (PDPK1), which delocalized actin fiber to destruct the $\mathrm{BBB}$ integrity. Intriguingly, unlike BBBprotective neuron-secreted miR-132, miR181c-containing extracellular vesicles can be secreted from brain metastatic cancer cells to break the $\mathrm{BBB}$, leading to brain metastasis (Tominaga et al., 2015).

Several miRNAs have been shown to regulate BBB integrity by targeting channels and transporters in brain endothelial cells. MiR-30a targets zinc transporter ZnT4, leading to reduced intracellular free zinc in endothelial cells and an increase in BBB permeability in both cellular and animal models of ischemic stroke (Wang et al., 2021). In contrast, in intracerebral hemorrhage, miR-27a-3p protects against $\mathrm{BBB}$ disruption by targeting endothelial aquaporin-11 (AQP11), a functional water channel that permeates both water and glycerol with a possible role in the pathophysiology of brain edema (Xi et al., 2018). This is a conflict with the reports showing miR-27a-3p is disruptive for endothelial barrier function (Young et al., 2013; Li et al., 2020), indicating that a specific miRNA may function differently via regulating different targets.

\section{CHALLENGES AND PERSPECTIVE}

The effect of miRNAs on BBB integrity makes them a promising target to transiently open the $\mathrm{BBB}$ for brain-targeted drug delivery and to restore $\mathrm{BBB}$ integrity for disease treatment. However, several key challenges remain to be overcome before translating BBB-targeted miRNA-based therapeutics into the clinic (Rupaimoole and Slack, 2017).

The first challenge is the identification of miRNA targets. Through the miRNA array, the change in miRNA expressions caused by disrupted BBB can be measured (Reijerkerk et al., 2013). However, how these significantly regulated miRNAs contribute to $\mathrm{BBB}$ disruption remains unclear. This requires the identification of the targets of these miRNAs. This process often includes experiments to measure mRNA or protein levels of possible targets after modulating endogenous miRNA expression. To further identify whether the regulation is direct or indirect, luciferase reporter assays have been commonly used ( $\mathrm{Li}$ et al., 2016). Because of the time for cloning and generation of mutants, these methods are quite time-consuming and only feasible for the identification of a small number of targets. In addition, it is becoming apparent that miRNAs may shift their targets in different types of cells and biological environments, further complicating the strategies for target validation.

The second challenge is the specificity of miRNA-based therapeutics. Each miRNA has dozens if not hundreds of potential targets. The ability of miRNAs to regulate a wide range of mRNAs gives them a unique advantage to regulate complex biological processes. However, it also raises possible side effects when miRNA expression is modulated. To overcome these drawbacks, antisense oligonucleotides (ASOs) that specifically block miRNA interaction with a mRNA of interest have been developed (Young et al., 2013; Zhao et al., 2017). Instead of modulating miRNA expression, these ASOs, named miRMask, target site blocker (TSB), or BlockmiRs, bind to the miRNA binding sites in the $3^{\prime}$ UTR of the target mRNA through full complementarity (Sonneville et al., 2017; Natarelli et al., 2018). Consequently, they prevent miRNA from regulating a specific mRNA. This can be highly useful in identifying the importance of a specific miRNA:mRNA interaction or developing miRNA therapeutics for validated drug targets. However, the design of TSB remains a challenge as the principles of the design are not fully understood. Further understanding and improvement in the design principles are required to improve the success rate.

The third challenge is the delivery of miRNA-based therapeutics to the brain. Current modifications of miRNA modulators, including mimics and inhibitors, have successfully increased their retention time in the circulation system (Rupaimoole and Slack, 2017). However, the majority of naked miRNA modulators are accumulated in the liver and kidneys. It is important to increase miRNA accumulation in the brain to enhance their effect targeting the BBB. Viral and non-viral delivery systems with specificity to brain endothelial cells have been successfully employed to deliver nucleotide-based drugs into the brain (Lee et al., 2019; Marcos-Contreras et al., 2020). However, the potential immunostimulatory effects and 
toxicity of these delivery systems may hinder clinical translation (Mitchell et al., 2021). Identification of highaffinity ligands targeting BBB-specific receptors and development of biocompatible delivery materials are required to improve the specificity and efficiency of BBB-targeted delivery systems.

\section{AUTHOR CONTRIBUTIONS}

JW and JL contributed to the conception and design of the review and wrote the first draft of the manuscript. $\mathrm{FX}, \mathrm{XZ}, \mathrm{XL}$, and YL critically revised the manuscript.

\section{REFERENCES}

Ayloo, S., and Gu, C. (2019). Transcytosis at the blood-brain barrier. Curr. Opin. Neurobiol. 57, 32-38. doi: 10.1016/0006-8993(87)90236-8

Bai, Y., Zhang, Y., Hua, J., Yang, X., Zhang, X., Duan, M., et al. (2016). Silencing microRNA-143 protects the integrity of the blood-brain barrier: implications for methamphetamine abuse. Sci. Rep. 6:35642.

Banks, W. A. (2016). From blood-brain barrier to blood-brain interface: new opportunities for CNS drug delivery. Nat. Rev. Drug Discov. 15, 275-292. doi: 10.1038/nrd.2015.21

Ben-Zvi, A., Lacoste, B., Kur, E., Andreone, B. J., Mayshar, Y., Yan, H., et al. (2014), Mfsd2a is critical for the formation and function of the blood-brain barrier. Nature 509, 507-511. doi: 10.1038/nature13324

Chakraborty, C., Sharma, A. R., Sharma, G., Bhattacharya, M., and Lee, S. S. (2020). MicroRNAs: possible regulatory molecular switch controlling the BBB microenvironment. Mol. Ther. Nucleic Acids 19, 933-936. doi: 10.1016/j.omtn. 2019.12.024

Cristante, E., McArthur, S., Mauro, C., Maggioli, E., Romero, I. A., WylezinskaArridge, M., et al. (2013). Identification of an essential endogenous regulator of blood-brain barrier integrity, and its pathological and therapeutic implications. Proc. Natl. Acad. Sci. U.S.A. 110, 832-841. doi: 10.1073/pnas.120936 2110

Demolli, S., Doddaballapur, A., Devraj, K., Stark, K., Manavski, Y., Eckart, A., et al. (2017). Shear stress-regulated miR-27b controls pericyte recruitment by repressing SEMA6A and SEMA6D. Cardiovasc. Res. 113, 681-691. doi: 10. 1093/cvr/cvx032

Fang, Z., He, Q. W., Li, Q., Chen, X. L., Baral, S., Jin, H. J., et al. (2016). MicroRNA150 regulates blood-brain barrier permeability via Tie- 2 after permanent middle cerebral artery occlusion in rats. FASEB J. 30, 2097-2107. doi: 10.1096/fj. 201500126

Fu, X., Niu, T., and Li, X. (2019). MicroRNA-126-3p attenuates intracerebral hemorrhage-induced blood-brain barrier disruption by regulating VCAM-1 expression. Front. Neurosci. 13:866. doi: 10.3389/fnins.2019.00866

Ge, X., Huang, S., Gao, H., Han, Z., Chen, F., Zhang, S., et al. (2016). miR-21$5 p$ alleviates leakage of injured brain microvascular endothelial barrier in vitro through suppressing inflammation and apoptosis. Brain Res. 1650, 31-40. doi: 10.1016/j.brainres.2016.07.015

Gebert, L. F. R., and MacRae, I. J. (2019). Regulation of microRNA function in animals. Nat. Rev. Mol. Cell Biol. 20, 21-37. doi: 10.1038/s41580-018-0045-7

Greene, C., Hanley, N., and Campbell, M. (2020). Blood-brain barrier associated tight junction disruption is a hallmark feature of major psychiatric disorders. Transl. Psychiatry 10:373.

Haruwaka, K., Ikegami, A., Tachibana, Y., Ohno, N., Konishi, H., Hashimoto, A., et al. (2019). Dual microglia effects on blood brain barrier permeability induced by systemic inflammation. Nat. Commun. 10:5816.

Juzwik, C. A., Sd, S., Zhang, Y., Paradis-Isler, N., Sylvester, A., Amar-Zifkin, A., et al. (2019). microRNA dysregulation in neurodegenerative diseases: a systematic review. Prog. Neurobiol. 182:101664. doi: 10.1016/j.pneurobio.2019.101664

Kozomara, A., Birgaoanu, M., and Griffiths-Jones, S. (2019). miRBase: from microRNA sequences to function. Nucleic Acids Res. 47, D155-D162.
All authors contributed to the article and approved the submitted version.

\section{FUNDING}

This work was partially supported by the Scientific Research Project of Hubei University of Science and Technology (No. 2019-21GP07 to JW), Research Project of Pharmacy Key Discipline of Hubei University of Science and Technology (No. 2019-20YZ15 to XL), and Open Fund Project of Hubei Key Laboratory of Cardiovascular, Cerebrovascular, and Metabolic Disorders (No. 2020TNB08 to YL).

Lampugnani, M. G., Dejana, E., and Giampietro, C. (2018). Vascular endothelial (VE)-cadherin, endothelial adherens junctions, and vascular disease. Cold Spring Harb. Perspect. Biol. 10:a029322. doi: 10.1101/cshperspect.a029322

Lee, S. W. L., Paoletti, C., Campisi, M., Osaki, T., Adriani, G., Kamm, R. D., et al. (2019). MicroRNA delivery through nanoparticles. J. Control. Release 313, 80-95. doi: 10.1016/j.jconrel.2019.10.007

Li, D., Liu, Y., Pei, C., Zhang, P., Pan, L., Xiao, J., et al. (2017). miR-285Yki/Mask double-negative feedback loop mediates blood-brain barrier integrity in Drosophila. Proc. Natl. Acad. Sci. U.S.A. 114, E2365-E2374.

Li, J., Zhao, Y., Choi, J., Ting, K. K., Coleman, P., Chen, J., et al. (2020). Targeting miR-27a/VE-cadherin interactions rescues cerebral cavernous malformations in mice. PLoS Biol. 18:e3000734. doi: 10.1371/journal.pbio.3000734

Li, J., Zhao, Y., Lu, Y., Ritchie, W., Grau, G., Vadas, M. A., et al. (2016). The Polycistronic miR-23-27-24 complexes target endothelial cell junctions: differential functional and molecular effects of miR-23a and miR-23b. Mol. Ther. Nucleic Acids 5:e354. doi: 10.1038/mtna.2016.62

Li, W., Chen, Z., Chin, I., Chen, Z., and Dai, H. (2018). The role of VEcadherin in blood-brain barrier integrity under central nervous system pathological conditions. Curr. Neuropharmacol. 16, 1375-1384. doi: 10.2174/ 1570159x16666180222164809

Liu, W., Cai, H., Lin, M., Zhu, L., Gao, L., Zhong, R., et al. (2016). MicroRNA-107 prevents amyloid-beta induced blood-brain barrier disruption and endothelial cell dysfunction by targeting Endophilin-1. Exp. Cell Res. 343, 248-257. doi: 10.1016/j.yexcr.2016.03.026

Liu, W., Wang, P., Shang, C., Chen, L., Cai, H., Ma, J., et al. (2014). Endophilin-1 regulates blood-brain barrier permeability by controlling ZO-1 and occludin expression via the EGFR-ERK1/2 pathway. Brain Res. 1573, 17-26. doi: 10. 1016/j.brainres.2014.05.022

Lopez-Ramirez, M. A., Wu, D., Pryce, G., Simpson, J. E., Reijerkerk, A., KingRobson, J., et al. (2014). MicroRNA-155 negatively affects blood-brain barrier function during neuroinflammation. FASEB J. 28, 2551-2565. doi: 10.1096/fj. 13-248880

Ludwig, N., Fehlmann, T., Kern, F., Gogol, M., Maetzler, W., Deutscher, S., et al. (2019). Machine learning to detect Alzheimer's disease from circulating noncoding RNAs. Genomics Proteomics Bioinformatics 17, 430-440. doi: 10.1016/j. gpb.2019.09.004

Ma, F., Sun, P., Zhang, X., Hamblin, M. H., and Yin, K. J. (2020). Endotheliumtargeted deletion of the miR-15a/16-1 cluster ameliorates blood-brain barrier dysfunction in ischemic stroke. Sci. Signal. 13:eaay5686. doi: 10.1126/scisignal. aay5686

Ma, Q., Dasgupta, C., Li, Y., Huang, L., and Zhang, L. (2017). MicroRNA210 suppresses junction proteins and disrupts blood-brain barrier integrity in neonatal rat hypoxic-ischemic brain injury. Int. J. Mol. Sci. 18:1356. doi: 10.3390/ijms18071356

Marcos-Contreras, O. A., Greineder, C. F., Kiseleva, R. Y., Parhiz, H., Walsh, L. R., Zuluaga-Ramirez, V., et al. (2020). Selective targeting of nanomedicine to inflamed cerebral vasculature to enhance the blood-brain barrier. Proc. Natl. Acad. Sci. U.S.A. 117, 3405-3414. doi: 10.1073/pnas.1912012117

Mishra, R., and Singh, S. K. (2013). HIV-1 Tat C modulates expression of miRNA-101 to suppress VE-cadherin in human brain microvascular 
endothelial cells. J. Neurosci. 33, 5992-6000. doi: 10.1523/jneurosci.4 796-12.2013

Mitchell, M. J., Billingsley, M. M., Haley, R. M., Wechsler, M. E., Peppas, N. A., and Langer, R. (2021). Engineering precision nanoparticles for drug delivery. Nat. Rev. Drug Discov. 20, 101-124.

Nag, S. (1995). Role of the endothelial cytoskeleton in blood-brain-barrier permeability to protein. Acta Neuropathol. 90, 454-460. doi: 10.1007/ s004010050355

Natarelli, L., Geissler, C., Csaba, G., Wei, Y., Zhu, M., di Francesco, A., et al. (2018). miR-103 promotes endothelial maladaptation by targeting lncWDR59. Nat. Commun. 9:2645.

Nation, D. A., Sweeney, M. D., Montagne, A., Sagare, A. P., D’Orazio, L. M., Pachicano, M., et al. (2019). Blood-brain barrier breakdown is an early biomarker of human cognitive dysfunction. Nat. Med. 25, 270-276. doi: 10. 1038/s41591-018-0297-y

Profaci, C. P., Munji, R. N., Pulido, R. S., and Daneman, R. (2020). The bloodbrain barrier in health and disease: important unanswered questions. J. Exp. Med. 217:e20190062.

Qian, Y., Chopp, M., and Chen, J. (2020). Emerging role of microRNAs in ischemic stroke with comorbidities. Exp. Neurol. 331:113382. doi: 10.1016/j.expneurol. 2020.113382

Reijerkerk, A., Lopez-Ramirez, M. A., van Het Hof, B., Drexhage, J. A., Kamphuis, W. W., Kooij, G., et al. (2013). MicroRNAs regulate human brain endothelial cell-barrier function in inflammation: implications for multiple sclerosis. J. Neurosci. 33, 6857-6863. doi: 10.1523/jneurosci.3965-12.2013

Rom, S., Dykstra, H., Zuluaga-Ramirez, V., Reichenbach, N. L., and Persidsky, Y. (2015). miR-98 and let- $7 g^{*}$ protect the blood-brain barrier under neuroinflammatory conditions. J. Cereb Blood Flow Metab. 35, 1957-1965. doi: $10.1038 /$ jcbfm.2015.154

Rupaimoole, R., and Slack, F. J. (2017). MicroRNA therapeutics: towards a new era for the management of cancer and other diseases. Nat. Rev. Drug Discov. 16, 203-222. doi: 10.1038/nrd.2016.246

Shigemori, Y., Katayama, Y., Mori, T., Maeda, T., and Kawamata, T. (2006). Matrix metalloproteinase- 9 is associated with blood-brain barrier opening and brain edema formation after cortical contusion in rats. Acta Neurochir. Suppl. 96, 130-133. doi: 10.1007/3-211-30714-1_29

Song, J., Hu, Y., Li, H., Huang, X., Zheng, H., Hu, Y., et al. (2018). miR1303 regulates BBB permeability and promotes CNS lesions following CA16 infections by directly targeting MMP9. Emerg. Microbes Infect. 7:155.

Sonneville, F., Ruffin, M., Coraux, C., Rousselet, N., Le Rouzic, P., Blouquit-Laye, S., et al. (2017). MicroRNA-9 downregulates the ANO1 chloride channel and contributes to cystic fibrosis lung pathology. Nat. Commun. 8:710.

Sonoda, T., Matsuzaki, J., Yamamoto, Y., Sakurai, T., Aoki, Y., Takizawa, S., et al. (2019). Serum MicroRNA-based risk prediction for stroke. Stroke 50, 1510-1518. doi: 10.1161/strokeaha.118.023648

Starhof, C., Hejl, A. M., Heegaard, N. H. H., Carlsen, A. L., Burton, M., Lilje, B., et al. (2019). The biomarker potential of cell-free microRNA from cerebrospinal fluid in Parkinsonian Syndromes. Mov. Disord. 34, 246-254. doi: 10.1002/mds. 27542

Sweeney, M. D., Ayyadurai, S., and Zlokovic, B. V. (2016). Pericytes of the neurovascular unit: key functions and signaling pathways. Nat. Neurosci. 19, 771-783. doi: 10.1038/nn.4288

Sweeney, M. D., Kisler, K., Montagne, A., Toga, A. W., and Zlokovic, B. V. (2018). The role of brain vasculature in neurodegenerative disorders. Nat. Neurosci. 21, 1318-1331. doi: 10.1038/s41593-018-0234-x

Taddei, A., Giampietro, C., Conti, A., Orsenigo, F., Breviario, F., Pirazzoli, V., et al. (2008). Endothelial adherens junctions control tight junctions by VEcadherin-mediated upregulation of claudin-5. Nat. Cell Biol. 10, 923-934. doi: $10.1038 /$ ncb 1752
Ting, K. K., Zhao, Y., Shen, W., Coleman, P., Yam, M., Chan-Ling, T., et al. (2019). Therapeutic regulation of VE-cadherin with a novel oligonucleotide drug for diabetic eye complications using retinopathy mouse models. Diabetologia 62, 322-334. doi: 10.1007/s00125-018-4770-4

Tominaga, N., Kosaka, N., Ono, M., Katsuda, T., Yoshioka, Y., Tamura, K., et al. (2015). Brain metastatic cancer cells release microRNA-181c-containing extracellular vesicles capable of destructing blood-brain barrier. Nat. Commun. 6:6716.

Toyama, K., Spin, J. M., Deng, A. C., Huang, T. T., Wei, K., Wagenhauser, M. U., et al. (2018). MicroRNA-mediated therapy modulating bloodbrain barrier disruption improves vascular cognitive impairment. Arterioscler. Thromb. Vasc. Biol. 38, 1392-1406. doi: 10.1161/atvbaha.118.31 0822

Treiber, T., Treiber, N., and Meister, G. (2019). Regulation of microRNA biogenesis and its crosstalk with other cellular pathways. Nat. Rev. Mol. Cell Biol. 20, 5-20. doi: 10.1038/s41580-018-0059-1

Turner, R. J., and Sharp, F. R. (2016). Implications of MMP9 for blood brain barrier disruption and hemorrhagic transformation following ischemic stroke. Front. Cell. Neurosci. 10:56. doi: 10.3389/fncel.2016.00056

Vanlandewijck, M., He, L., Mae, M. A., Andrae, J., Ando, K., Del Gaudio, F., et al. (2018). A molecular atlas of cell types and zonation in the brain vasculature. Nature 554, 475-480. doi: 10.1038/nature25739

Wang, P., Pan, R., Weaver, J., Jia, M., Yang, X., Yang, T., et al. (2021). MicroRNA30a regulates acute cerebral ischemia-induced blood-brain barrier damage through ZnT4/zinc pathway. J. Cereb. Blood Flow Metab. 41, 641-655. doi: $10.1177 / 0271678 \times 20926787$

Xi, T., Jin, F., Zhu, Y., Wang, J., Tang, L., Wang, Y., et al. (2018). miR-27a$3 \mathrm{p}$ protects against blood-brain barrier disruption and brain injury after intracerebral hemorrhage by targeting endothelial aquaporin-11. J. Biol. Chem. 293, 20041-20050. doi: 10.1074/jbc.ra118.001858

Xu, B., Zhang, Y., Du, X. F., Li, J., Zi, H. X., Bu, J. W., et al. (2017). Neurons secrete miR-132-containing exosomes to regulate brain vascular integrity. Cell Res. 27, 882-897. doi: 10.1038/cr.2017.62

Young, J. A., Ting, K. K., Li, J., Moller, T., Dunn, L., Lu, Y., et al. (2013). Regulation of vascular leak and recovery from ischemic injury by general and VE-cadherinrestricted miRNA antagonists of miR-27. Blood 122, 2911-2919. doi: 10.1182/ blood-2012-12-473017

Zhang, T., Tian, C., Wu, J., Zhang, Y., Wang, J., Kong, Q., et al. (2020). MicroRNA182 exacerbates blood-brain barrier (BBB) disruption by downregulating the mTOR/FOXO1 pathway in cerebral ischemia. FASEB J. 34, 13762-13775. doi: 10.1096/fj.201903092r

Zhao, Y., Ting, K., Li, J., Cogger, V. C., Chen, J., Johansson-Percival, A., et al. (2017). Targeting vascular endothelial-cadherin in tumor-associated blood vessels promotes T cell-mediated immunotherapy. Cancer Res. 77, 4434-4447. doi: 10.1158/0008-5472.can-16-3129

Zuo, X., Lu, J., Manaenko, A., Qi, X., Tang, J., Mei, Q., et al. (2019). MicroRNA-132 attenuates cerebral injury by protecting blood-brain-barrier in MCAO mice. Exp. Neurol. 316, 12-19. doi: 10.1016/j.expneurol.2019.03.017

Conflict of Interest: The authors declare that the research was conducted in the absence of any commercial or financial relationships that could be construed as a potential conflict of interest.

Copyright (C) 2021 Wang, Xu, Zhu, Li, Li and Li. This is an open-access article distributed under the terms of the Creative Commons Attribution License (CC BY). The use, distribution or reproduction in other forums is permitted, provided the original author(s) and the copyright owner(s) are credited and that the original publication in this journal is cited, in accordance with accepted academic practice. No use, distribution or reproduction is permitted which does not comply with these terms. 